\title{
Reliability Optimization of Entropy Based Series-Parallel System Using Global Criterion Method
}

\author{
G. S. MAHAPATRA \\ Department of Engineering Sciences and Humanities, Siliguri Institute of Technology, Siliguri, India \\ Email:g_s_mahapatra@yahoo.com
}

\begin{abstract}
In this paper, we have considered a series-parallel system to find out optimum system reliability with an additional entropy objective function. Maximum system reliability of series-parallel system is depending on proper allocation of redundancy component in different stage. The goal of entropy based reliability redundancy allocation problem is to find optimal number of redundancy component in each stage such a manner that maximize the system reliability subject to available total system cost. Global criterion method is used to analyze entropy based reliability optimization problem with different weight function of objective functions. Numerical examples have been provided to illustrate the model.
\end{abstract}

Keywords: reliability, series-parallel system, redundancy, entropy, global criterion method

\section{Introduction}

The problem of reliability redundancy allocation is to find out optimal system reliability by optimal allocation of redundancy components in series-parallel system. Reliability of a multi-stage system can be improved by adding similar or some different components redundancy to each sub-system as design alternatives. The design of a reliable system was improved by Hikita et al. [3] by the addition of redundant components. Several researcher [1, $2,4,5,12,13,17,20-22]$ presented redundancy optimization with multiple objective functions of system reliability, system cost and system weight etc. and solve that objective redundancy allocation problem by different algorithm and nonlinear optimization techniques for multiobjective system reliability design optimization in fuzzy and crisp environments. Tillman et al. [19] presented a comprehensive survey of previous works for system reliability with redundancy. Singh and Misra [18], Kuo and Prasad [7], Kuo et al. [8], and Misra [10] presented reliability redundant allocation problem to increase the system reliability, which is important in reliability engineering.

For series-parallel reliability redundancy allocation problem, entropy represents the lack of the information about the state of the each sub-system. Very few authors discussed reliability analysis with entropy consideration. Musto and Saridis [11] presented entropy-based reliability assessment technique. The technique was demonstrated in a case study of a robotic system. Rocchi [15] introduced the entropy function in order to study the reliability and repairability of systems. Ridder [14] inves- tigated the application and usability of the cross-entropy method for rare event simulation in Markovian reliability models. Rocchi [16] discussed and calculated the reliability function during system again through the stochastic entropy. Kroese et al. [6] introduced a new approach based on cross-entropy method for optimization of network reliability.

Here, we have considered a multi-objective entropy based reliability redundancy problem to finding the optimum number of redundant components, which maximize the system reliability with entropy as an additional objective function subject to available system cost. The redundancy reliability optimization problem is considered with two objective functions such as maximum system reliability and maximum entropy amount simultaneously with restriction on system cost. Numerical example is presented using global criterion method.

\section{Reliability Redundancy Allocation Model}

\subsection{Notations}

Series Parallel system, reliability redundancy allocation model is developed under the following notations.

$\mathrm{R}_{\mathrm{i}}$ reliability of each component of reliability model in the $i^{\text {th }}$ stage,

$\mathrm{C}_{\mathrm{i}}$ cost of each component of reliability model in the $\mathrm{i}^{\text {th }}$ stage,

$\mathrm{C}$ available system cost of the reliability model,

$x_{i}$ number of redundancy components in the $\mathrm{i}^{\text {th }}$ stage (decision variables), 
$R_{s}\left(x_{1}, x_{2}, \ldots, x_{n}\right)$ system reliability function of the reliability model,

$C_{s}\left(x_{1}, x_{2}, \ldots, x_{n}\right) \quad$ system cost function of the reliability model,

$\operatorname{En}\left(x_{1}, x_{2}, \ldots, x_{n}\right) \quad$ entropy function of the reliability model.

\subsection{Reliability Redundancy Allocation Problem}

It is to be considered that an $\mathrm{n}$ stage series system and at each stage added $\left(\mathrm{x}_{\mathrm{i}}-1\right)$ redundant components in parallel, the objectives are to determine the number of redundant components at each stage such that the system reliability will be maximize subject to related cost constraints.

Therefore the maximization of $\mathrm{R}_{\mathrm{s}}$ subject to the limited available cost $\mathrm{C}$ has to be found.

Therefore the problem becomes

$$
\operatorname{Max}_{s}\left(x_{1}, x_{2}, \ldots, x_{n}\right)=\prod_{i}^{n}\left\{1-\left(1-R_{i}\right)^{x_{i}}\right\}
$$

subject to

$$
\begin{gathered}
C_{s}\left(x_{1}, x_{2}, \ldots, x_{n}\right)=\sum_{i=1}^{n} C_{i}\left(x_{i}+\exp \left(x_{i} / 4\right)\right) \leq C \\
\mathrm{x}_{\mathrm{i}}>1 \text { for } \mathrm{i}=1,2, \ldots, \mathrm{n} .
\end{gathered}
$$

\subsection{Entropy in Series Parallel System}

Entropy has important physical implications as the amount of "disorder" of a system. A more abstract definition is used in mathematics.

The Shannon entropy of a variable $\mathrm{X}$ is defined as

$$
E n(X)=-\sum_{x} p(x) \ln p(x)
$$

where $\mathrm{p}(\mathrm{x})$ is the probability that $\mathrm{X}$ is the state $\mathrm{x}$, and $\mathrm{p}(\mathrm{x})$ $\log \mathrm{p}(\mathrm{x})$ is defined as 0 if $\mathrm{p}(\mathrm{x})=0$.

Reliability redundancy allocation problem is the redundancy distribution of each stage of a series-parallel system. To determine a suitable measure of allocation, let us consider a n-stage series-parallel system with $\mathrm{x}_{\mathrm{i}}$ $(i=1,2, \ldots, n)$ number of redundant component of each $i^{\text {th }}$ stage of the system. It is known that $\mathrm{x}_{\mathrm{i}}$ are positive integer and total number of components is $\sum_{i} x_{i}$. Redundancy allocation of components share of $i^{\text {th }}$ stage is the share of the total number of redundant component is $p_{i}=\frac{x_{i}}{\sum x_{i}}$. Normalizes the redundancy numbers $\mathrm{x}_{\mathrm{i}}$ by dividing them by the total number of redundant components $\sum_{i} x_{i}$ then the probability distribution $p_{i}=\frac{x_{i}}{\sum_{i} x_{i}}$ is found.

The measure of allocation shall be defined as the expected information of the message which transforms the system shares into the share of each stage.

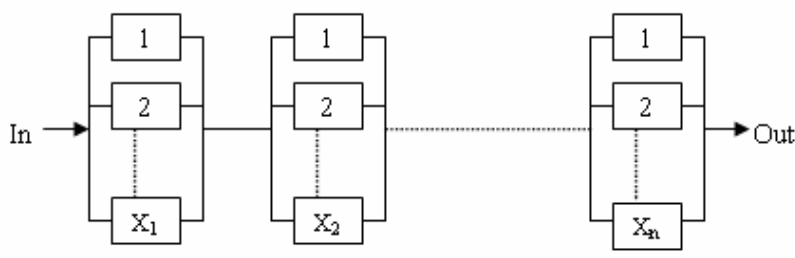

Figure 1. A schematic diagram of the n-stage system

$$
\begin{array}{r}
\text { So } \operatorname{En}\left(x_{1}, x_{2}, \ldots, x_{n}\right)=-\sum_{i=1}^{n} p_{i} \ln p_{i} \\
\text { where } p_{i}=\frac{x_{i}}{\sum_{i} x_{i}}
\end{array}
$$

The each stage share $p_{i}=\frac{x_{i}}{\sum_{i} x_{i}}$ satisfying the condition $p_{i} \geq 0(\forall \mathrm{i}=1,2, \ldots, \mathrm{n})$ and $\sum_{i} p_{i}=1$ defines a probability distribution and the Shannon-entropy measure the diversity of the probability distribution $\left\{p_{1}, p_{2}, \ldots, p_{n}\right\}$. Maximum is reached when $p_{1}=p_{2}=\ldots=p_{n}=1 / n$ i.e. when allocation of all stage have the same no of redundant components. Since increasing of $\mathrm{x}_{\mathrm{i}}$, maximizing $\operatorname{In} p_{i}$ is equivalent to maximizing entropy as defined above. This is one of the reasons why the entropy optimization model is particularly suitable for the redundancy allocation problem. In redundancy allocation problem, entropy acts as a measure of dispersal of allocation between stages. So it will be more potential if we would like to have maximum system reliability as well as maximize entropy measure.

\subsection{Multi-Objective Entropy Redundancy Allocation Problem}

Taking entropy function as additional objective function the problem (1) becomes

$$
\begin{aligned}
& \text { Maximize } R_{s}\left(x_{1}, x_{2}, \ldots, x_{n}\right)=\prod_{i}^{n}\left\{1-\left(1-\tilde{R}_{i}\right)^{x_{i}}\right\} \\
& \text { Maximize } \operatorname{En}\left(x_{1}, x_{2}, \ldots, x_{n}\right)=-\sum_{i=1}^{n}\left(x_{i} / \sum x_{i}\right) \log \left(x_{i} / \sum x_{i}\right)
\end{aligned}
$$

Subject to same constraint and restriction as in (1)

\section{Method of Global Criterion}

A multi-objective non-linear problem may be taken in the following form

Maximize/Minimize $\mathrm{f}(\mathrm{x})=\left[\mathrm{f}_{1}(\mathrm{x}), \mathrm{f}_{2}(\mathrm{x}), \ldots, \mathrm{f}_{\mathrm{k}}(\mathrm{x})\right]^{\mathrm{T}}(5)$

subject to $x \in X=\left\{x \in R^{n}: g_{j}(x) \leq b_{j}\right.$ for $j=1, \ldots, m$; $\mathrm{l}_{\mathrm{i}} \leq \mathrm{x}_{\mathrm{i}} \leq \mathrm{u}_{\mathrm{i}}$ for $\left.\mathrm{i}=1,2, \ldots, \mathrm{n}\right\}$.

Solve the multi-objective non-linear problem (5) as a 
single objective non-linear problem $\mathrm{k}$ times for each problem by taking one of the objective at a time and ignoring the others. From the result, determine the corresponding values for every objective for each derived solution. For each objective $f_{r}(x)$, find lower bound (minimum) $f_{r}^{l}$ and the upper bound (maximum) $f_{r}^{u}$.

In the global criterion method [9], the distance between some reference point and the feasible objective region is minimized. The analyst has to select the reference point and the metric for measuring the distances. Suppose that the weighting coefficients $w_{r}$ are real numbers such that $w_{r} \geq 0, \forall r=1,2, \ldots, k$ and $\sum_{r=1}^{k} w_{r}=1$.

Here we examine the method where the ideal objective vector is used as a reference point and $L_{p}$-metrics are used for measuring. In this case, the weighted $L_{p}$ -problem for minimizing distances is stated as

$$
\text { Minimize } L_{p}(f(x))=\left(\sum_{r=1}^{m} w_{r}\left|\frac{f_{r}(x)-f_{r}^{l}}{f_{i}^{u}-f_{r}^{l}}\right|^{p}\right)^{1 / p}
$$

\section{Subject to $x \in X$ for $1 \leq p<\infty$}

The exponent $1 / \mathrm{p}$ may be dropped. Problems with or without the exponent $1 / \mathrm{p}$ are equivalent for $1 \leq p<\infty$. The solution obtained depends greatly on the value chosen for $\mathrm{p}$, commonly used choices are $\mathrm{p}=1,2$ or $\infty$.

$$
\text { For } \mathrm{p}=1, \quad L_{1}(f(x))=\sum_{r=1}^{m} w_{r}\left|\frac{f_{r}(x)-f_{r}^{l}}{f_{r}^{u}-f_{r}^{l}}\right|
$$

The objective function $L_{1}(f(x))$ is the sum of the normalized weighted deviations, which is to be minimized.

$$
\text { For } \mathrm{p}=2, \quad L_{2}(f(x))=\left(\sum_{r=1}^{m} w_{r}\left|\frac{f_{r}(x)-f_{r}^{l}}{f_{r}^{u}-f_{r}^{l}}\right|^{2}\right)^{1 / 2}
$$

When $p$ becomes larger, the minimization of the deviation becomes more and more important.

If $p=\infty, \operatorname{GCM}(6)$ is of the form

$$
\text { Minimize } \max _{r=1,2, \ldots, k} w_{r}\left|\frac{f_{r}(x)-f_{r}^{l}}{f_{r}^{u}-f_{r}^{l}}\right|
$$

Subject to $x \in X$.

The problem (9) can be transformed into the following form

$$
\text { Minimize } \lambda
$$

Subject to $w_{r}\left|\frac{f_{r}(x)-f_{r}^{l}}{f_{r}^{u}-f_{r}^{l}}\right| \leq \lambda$ for all $\mathrm{r}=1,2, \ldots, \mathrm{k}$. $x \in X$ where both $x \in \mathfrak{R}^{n}$ and $\lambda \in \mathfrak{R}$ are variables.

\section{Global Criterion Method on Entropy Based Reliability Redundancy Problem}

In entropy based reliability optimization of series-parallel system, maximum system reliability $R_{s}(x)$ and maximum entropy $E n(x)$ has to be found, having subject to the system cost constraint $C_{s}(x)$ with goal of system cost is $C$. So the problem is a multi-objective entropy reliability redundancy allocation problem as follows

$$
\begin{gathered}
\text { Maximize } R_{s}(x) \\
\text { Maximize } \operatorname{En}(x)
\end{gathered}
$$

Subject to $C_{s}(x) \leq C$

Where $x=\left(x_{1}, x_{2}, \ldots, x_{n}\right)$ and $\mathrm{x}_{\mathrm{i}}>1$ for $\mathrm{i}=1,2, \ldots, \mathrm{n}$.

To solve the above multi-objective reliability optimization problem (11), according to section 3 pay-off matrix is formulated as follows:

$$
\begin{aligned}
& R_{s}(x) \quad E n(x) \\
& x^{1}\left[\begin{array}{cc}
R_{s}^{*}\left(x^{*}\right) & E n(x) \\
R_{s}(x) & E n^{*}\left(x^{*}\right)
\end{array}\right]
\end{aligned}
$$

Now lower and upper bounds of $R_{s}(x)$ and $E n(x)$ are identified and denoted as $R_{s}^{L}, R_{s}^{U}$ and $E n^{L}, E n^{U}$ respectively.

Using Global criterion method for the problem (11), the weighted $L_{p}$-problem for minimizing distances is stated as

Minimize

$$
\begin{aligned}
& L_{p}\left(R_{s}(x), E n(x)\right)= \\
& \left(w_{1}\left|\frac{R_{s}(x)-R_{s}^{L}}{R_{s}^{U}-R_{s}^{L}}\right|^{p}+w_{2}\left|\frac{E n(x)-E n^{L}}{E n^{U}-E n^{L}}\right|^{p}\right)^{1 / p}
\end{aligned}
$$

Subject to $C_{s}(x) \leq C$, for $1 \leq p<\infty$

Putting different value of $\mathrm{p}(1,2$ or $\infty)$ in (12), we get as follows

$$
\begin{aligned}
& \text { For } \mathrm{p}=1, \\
& L_{1}\left(R_{s}(x), E n(x)\right) \\
& =w_{1}\left|\frac{R_{s}(x)-R_{s}^{L}}{R_{s}^{U}-R_{s}^{L}}\right|+w_{2}\left|\frac{E n(x)-E n^{L}}{E n^{U}-E n^{L}}\right|
\end{aligned}
$$

For $\mathrm{p}=2$,

$$
\begin{aligned}
& L_{2}\left(R_{s}(x), E n(x)\right)= \\
& \left(w_{1}\left|\frac{R_{s}(x)-R_{s}^{L}}{R_{s}^{U}-R_{s}^{L}}\right|^{2}+w_{2}\left|\frac{E n(x)-E n^{L}}{E n^{U}-E n^{L}}\right|^{2}\right)^{1 / 2}
\end{aligned}
$$


For $p=\infty,(12)$ is of the form

Minimize $\lambda$

$$
\begin{gathered}
\text { Subject to } \quad w_{1}\left|\frac{R_{s}(x)-R_{s}^{L}}{R_{s}^{U}-R_{s}^{L}}\right| \leq \lambda \\
w_{2}\left|\frac{E n(x)-E n^{L}}{E n^{U}-E n^{L}}\right| \leq \lambda \\
\lambda \in \Re .
\end{gathered}
$$

To solve the entropy based reliability redundancy allocation problem (11) using GCM, we have to solve (13), (14), (15) with same constraints as in Equation (10) for different weight.

\section{Numerical Example}

A four stage reliability redundancy allocation problem with entropy objective function with cost constraints is considered for numerical exposure. The problem becomes as follows:

$$
\text { Maximize } R_{s}\left(x_{1}, x_{2}, x_{3}, x_{4}\right)=\prod_{i}^{4}\left\{1-\left(1-R_{i}\right)^{x_{i}}\right\}
$$

Maximize $E n\left(x_{1}, x_{2}, x_{3}, x_{4}\right)=-\sum_{i=1}^{4}\left(x_{i} / \sum x_{i}\right) \log \left(x_{i} / \sum x_{i}\right)$ (16) subject to

$$
C_{s}\left(x_{1}, x_{2}, \ldots, x_{n}\right)=\sum_{i=1}^{n} C_{i}\left(x_{i}+\exp \left(x_{i} / 4\right)\right) \leq C,
$$

$x_{i}>1$ for $i=1,2,3,4$.

Input parameters of the problem (5.1) are given in table 1 .

\section{Solution:}

Table 1. Input data for model (4)

\begin{tabular}{lllllllll}
\hline $\mathrm{R}_{1}$ & $\mathrm{R}_{2}$ & $\mathrm{R}_{3}$ & $\mathrm{R}_{4}$ & $\mathrm{C}_{1}$ & $\mathrm{C}_{2}$ & $\mathrm{C}_{3}$ & $\mathrm{C}_{4}$ & $\mathrm{C}$ \\
0.85 & 0.9 & 0.8 & 0.95 & 8 & 9 & 7 & 10 & 200 \\
\hline
\end{tabular}

The pay-off matrix is formulated as follows:

$$
\begin{aligned}
& R_{s}(x) \quad \operatorname{En}(x)
\end{aligned}
$$

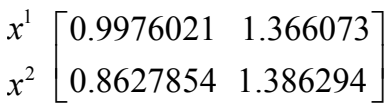

Here $R_{s}^{U}=0.9976021, R_{s}^{L}=0.8627854$, $E n^{U}=1.386294, E n^{L}=1.366073$ are identified and using these bounds construct the objective functions. The optimal solutions of the multi-objective reliability optimization model (16) using global criterion method (following (13), (14) and (15)) are given in Table 2 for different preference values of the objective functions.

In case-I, Table 2 shows different optimal solutions when the decision maker supplies more preference to the entropy function than the reliability function. Here $R_{s}^{*}\left(x^{*}\right)$ is maximum when $\mathrm{p}=2$, whereas $\operatorname{En}^{*}\left(x^{*}\right)$ is maximum when $p=2$ or $\infty$.

In Table 2, case-II gives different optimal solutions when the decision maker supplies equal preferences to the reliability function and entropy function. Here $R_{s}^{*}\left(x^{*}\right)$ is maximum when $\mathrm{p}=1$, whereas $\operatorname{En}^{*}\left(x^{*}\right)$ is remains unaltered for $\mathrm{p}$.

\begin{tabular}{|c|c|c|c|c|c|c|c|c|c|}
\hline Case & $\mathrm{w}_{1}$ & $\mathrm{w}_{2}$ & $\mathrm{p}$ & $x_{1}^{*}$ & $x_{2}^{*}$ & $x_{3}^{*}$ & $x_{4}^{*}$ & $R_{S}^{*}\left(x^{*}\right)$ & $E n^{*}\left(x^{*}\right)$ \\
\hline & & & 1 & 2 & 3 & 2 & 3 & 0.9373444 & 1.366159 \\
\hline \multirow[t]{3}{*}{ I } & 0.2 & 0.8 & 2 & 2 & 2 & 3 & 2 & 0.9575832 & 1.368922 \\
\hline & & & $\infty$ & 2 & 3 & 2 & 2 & 0.9351179 & 1.368922 \\
\hline & & & 1 & 2 & 2 & 3 & 2 & 0.9575832 & 1.368922 \\
\hline \multirow[t]{3}{*}{ II } & 0.5 & 0.5 & 2 & 2 & 2 & 2 & 3 & 0.9288999 & 1.368922 \\
\hline & & & $\infty$ & 2 & 2 & 2 & 3 & 0.9288999 & 1.368922 \\
\hline & & & 1 & 2 & 2 & 2 & 2 & 0.9266935 & 1.386294 \\
\hline \multirow[t]{2}{*}{ III } & 0.8 & 0.2 & 2 & 2 & 2 & 2 & 2 & 0.9266935 & 1.386294 \\
\hline & & & $\infty$ & 2 & 2 & 2 & 2 & 0.9266935 & 1.386294 \\
\hline
\end{tabular}

In case-III, Table 2 shows different optimal solutions when the decision maker supplies more preference to the reliability function than the entropy function. Here $R_{s}^{*}\left(x^{*}\right)$ and $E n^{*}\left(x^{*}\right)$ remains unaltered for $\mathrm{p}$.

Table 2. Optimal solution for different weigtages of system reliability $\left(w_{1}\right)$ and entropy functions $\left(w_{2}\right)$ by $G_{C M}$ 


\section{Conclusions}

Here redundancy allocation problem of series-parallel system with reliability and entropy objectives is presented. Global criterion method is used to solve the problem of multi-objective reliability redundancy allocation problem with entropy function. Two objective functions are combined into a single objective function by the global criterion method. The optimal solutions for different preferences on objective functions are presented. Decision-maker may obtain the Pareto optimal results according to his expectation of system cost.

Here it is considered that the problem as to finding the optimum number of redundancies, which maximize the system reliability and entropy subject to the limited system cost. The system reliability increases with increases of redundancies and entropy of the system decreases which is expected.

\section{REFERENCES}

[1] A. O. Charles Elegbede, C. Chu, K. H. Adjallah, and F. Yalaoui, "Reliability allocation through cost minimization," IEEE Transactions on Reliability, Vol. 52, No. 1, pp. 106-111. 2003.

[2] A. K. Dhingra, "Optimal apportionment of reliability \& redundancy in series systems under multiple objectives," IEEE Transactions on Reliability, Vol. 41, No. 4, pp. 576-582, 1992.

[3] M. Hikita, Y. Nakagawa, K. Nakashima, and H. Narihisa, "Reliability optimization of system by a surrogate- constraints algorithm," IEEE Transactions on Reliability, Vol R-41, pp. 473-480, 1992.

[4] Y. C. Hsieh, "A linear approximation for redundant reliability problems with multiple component choices," Computer \& Industrial Engineering, Vol. 44, pp. 91-103, 2003.

[5] H. Z. Huang, "Fuzzy multi-objective optimization decision-making of reliability of series system," Microelectronic and Reliability, Vol. 37, No. 3, pp. 447-449, 1997.

[6] D. P. Kroese, K. P. Hui, and S. Nariai, "Network reliability optimization via the cross-entropy method," IEEE Transactions on Reliability, Vol. 56, No. 2, pp. 275-287. 2007.

[7] W. Kuo and V. R. Prasad, "An annotated overview of system reliability optimization," IEEE Transactions on Reliability, Vol. 49, No. 2, pp. 76-187, 2000.

[8] W. Kuo, V. R. Prasad, F. A. Tillman, and C. Hwang "Optimal reliability design-fundamentals and applications,"
Cambridge University Press, The Pitt Bulding, Trumpington Street, Cambridge, United Kingdom, 2001.

[9] K. Miettinen, "Nonlinear multiobjective optimization," Kluwer Academic Publishers, USA, 1999.

[10] K. B. Misra, "Multicriteria redundancy optimization using an efficient search procedure," International Journal of Systems Science, Vol. 22, No. 11, pp. 2171-2183. 1991.

[11] J. C. Musto and G. N. Saridis, "Entropy-based reliability analysis for intelligent machines," IEEE Transactions on Systems, Man, and Cybernetics, Part B, Vol. 27, No. 2, pp. 239-244, 1997.

[12] Pandey MK, Tiwari MK, Zuo MJ. Interactive enhanced particle swarm optimization: a multi-objective reliability application. Journal of Risk and Reliability 2007; 221(3); 177-191.

[13] S. S. Rao and A. K. Dhingra, "Reliability and redundancy apportionment using crisp and fuzzy multi-objective optimization approaches," Reliability Engineering and Systems Safety, Vol. 37, pp. 253-261, 1992.

[14] A. D. Ridder, "Importance sampling simulations of Markovian reliability systems using cross-entropy," Annals of Operations research, Vol. 134, pp. 119-136, 2005.

[15] P. Rocchi, "Boltzmann-like entropy in reliability theory," Entropy, Vol. 4, pp. 142-150, 2002.

[16] P. Rocchi, "Calculations of system aging through the stochastic entropy," Entropy, Vol. 8, pp. 134-142, 2006.

[17] M. Sakawa, "Multi-objective optimization by the surrogate worth trade-off method," IEEE Transactions on Reliability, Vol. R-27, pp. 311-314, 1978.

[18] H. Sinha and N. Misra, "On redundancy allocation in systems," Journal of Applied Probability, Vol. 31, pp. 1004-1014, 1994.

[19] F. A. Tillman, C. L. Hwang, and W. Kuo, "Optimization techniques for systems reliability with redundancy - A review," IEEE Transactions on Reliability, Vol. R-26, pp. 148-155, 1977.

[20] B. J. Yum and J. H. Kim, "Multi-level redundancy optimization in series systems," Computer \& Industrial Engineering, Vol. 46, pp. 337-346, 2004.

[21] R. Zhao and B. Liu, "Stochastic programming models for general redundancy-optimization problems," IEEE Transactions on Reliability, Vol. 52, No. 2, pp. 181-191, 2003.

[22] E. Zio, F. D. Maio, and S. Martorell, "Fusion of artificial neural networks and genetic algorithms for multi- objective system reliability design optimization," Journal of Risk and Reliability, Vol. 222, No. 2, pp. 115-226, 2008. 